\title{
30-day hospital readmission of older adults using care transitions after hospitalization: a pilot prospective cohort study
}

This article was published in the following Dove Press journal:

Clinical Interventions in Aging

17 June 2013

Number of times this article has been viewed

\author{
Paul Y Takahashi' \\ Lindsey R Haas ${ }^{2}$ \\ Stephanie M Quigg' \\ Ivana T Croghan' \\ James M Naessens ${ }^{2}$ \\ Nilay D Shah ${ }^{2}$ \\ Gregory J Hanson' \\ 'Division of Primary Care Internal \\ Medicine, Department of Medicine, \\ ${ }^{2}$ Department of Health Sciences \\ Research, Mayo Clinic, Rochester, \\ MN, USA
}

Purpose: Patients leaving the hospital are at increased risk of functional decline and hospital readmission. The Employee and Community Health service at Mayo Clinic in Rochester developed a care transition program (CTP) to provide home-based care services for medically complex patients. The study objective was to determine the relationship between CTP use, 30-day hospital readmission, and Emergency Room (ER) visits for adults over 60 years with high Elder Risk Assessment scores.

Patients and methods: This was a pilot prospective cohort study that included 20 patients that used the CTP and 20 patients discharged from the hospital without using the CTP. The medically complex study patients were drawn from the department of Employee and Community Health population between October 14, 2011 and September 27, 2012. The primary outcomes were 30-day hospital readmission or ER visit after discharge from the hospital. The secondary outcomes were within-group changes in grip strength, gait speed, and quality of life (QOL). Patients underwent two study visits, one at baseline and one at 30 days postbaseline. The primary analysis included time-to-event from baseline to rehospitalization or ER visit. Paired $t$-tests were used for secondary outcomes, with continuous scores.

Results: Of the 40 patients enrolled, 36 completed all study visits. The 30 -day hospital readmission rates for usual care patients were $10.5 \%$ compared with no readmissions for CTP patients. There were $31.6 \%$ ER visits in the UC group and $11.8 \%$ in the CTP group $(P=0.37)$. The secondary analysis showed some improvement in physical QOL scores (pre: 32.7 ; post: 39.4 ) for the CTP participants $(P<0.01)$ and no differences in gait speed or grip strength.

Conclusion: Based on this pilot study of care transition, we found nonsignificant lower hospital and ER utilization rates and improved physical QOL scores for patients in the CTP group. However, the data leads us to recommend future studies with larger sample sizes $(\mathrm{N}=250)$.

Keywords: case management, cohort study, frailty, geriatrics

\section{Introduction}

The care of patients and possible rehospitalization after a hospital stay has always been of the utmost importance to medical providers. All parties recognize the challenges of hospitalization. Older adults are often discharged from the hospital while still recuperating from an illness and still suffering functional disability. Ongoing illnesses can lead to further functional decline after hospitalization. With decline, many patients suffer one or multiple readmissions to the hospital. ${ }^{1}$ Up to half of readmissions may be preventable. ${ }^{2}$ In addition to other areas of focus to prevent rehospitalization, the time of discharge from hospital to home is a key transition in medical care and is the focus of quality improvement to prevent rehospitalization. ${ }^{3,4} \mathrm{~A}$ comprehensive
Correspondence: Paul Y Takahashi Mayo Clinic, 200 First Street SW, Rochester, MN 55905, USA

$\mathrm{Tel}+\mathrm{I} 50728425$ I I

Fax +I 5072660036

Email takahashi.paul@mayo.edu 
hospital discharge program can also support patients after their return home. This discharge process involves continued management of chronic diseases and ensures the completion of further tests and consultations. Ensuring the continuity of medical care may provide better outcomes. ${ }^{5}$ Patients without timely follow-up with a primary care provider have a tenfold higher risk of rehospitalization. ${ }^{6}$ Thus, a transition care program after hospitalization involves timely follow-up to ensure continuity, discussion of the diagnosis and hospital stay, and confirmation of the medication plan. ${ }^{7}$ This care planning often includes medication reconciliation and management, further follow-up, proper access, and communication if changes in condition occur. This is important to ensure adherence to medications and an understanding of those medications. A previous study of 162 patients showed a problem with medication reconciliation in $71 \%$ of discharge summaries. ${ }^{8}$ Thus, medication reconciliation can become an important aspect of care during transitions. ${ }^{9}$

One strategy of implementing a comprehensive medical care plan with medication reconciliation involves using a care transition team. Whereas these teams may vary, the principle involves a comprehensive discharge process and a timely follow-up visit. ${ }^{10}$ This posthospital follow-up visit often occurs within the home. This visit can be performed by a physician, nurse practitioner (NP), or registered nurse $(\mathrm{RN})$. The Employee and Community Health $(\mathrm{ECH})$ service at Mayo Clinic in Rochester, MN, USA implemented a care transition program (CTP), using an NP visit clinical practice model. An NP visited the home of high-risk patients within 1 to 3 days of their discharge from the hospital, to assess patients for clinical changes, to assess the home situation, and to improve patient education and communication. Nationally, the most comprehensive effort to improve the discharge process is the project RED (Re-Engineered Discharge), coordinated through the Agency for Healthcare Quality and Research. ${ }^{11}$ There has been other pioneering work in care transition, by Naylor et al and by Coleman et al. ${ }^{12,13}$ While these programs have shown positive results, a wider effort to implement quality improvement in the reduction of rehospitalization has not shown as robust results. ${ }^{14} \mathrm{~A}$ recent meta-analysis emphasized the importance of the care transition approach but also found the evidence in support of this to be lacking in some areas. ${ }^{15}$ We sought to determine the relationship between CTP services and time to hospital readmission within 30 days, the number of Emergency Room (ER) visits, functional status, and quality of life (QOL). To this end, we conducted a pilot prospective cohort study of medically complex patients who were recently dismissed from the hospital.

\section{Material and methods \\ Study design}

This was a prospective cohort study. The study was approved by the Mayo Clinic Institutional Review Board. Patients provided written informed consent for enrollment into the study. The study was conducted from October 14, 2011 to September 27, 2012.

\section{Setting}

The study was performed at the ECH department at Mayo Clinic in Rochester, MN, USA. The ECH is a primary care practice within a tertiary care center with four care sites, including one downtown practice, a rural practice, and two suburban practices. In 2011, ECH enrolled in the Minnesota Medical Home program, the aim of which is to improve the coordination of care for all primary care patients (with a special emphasis on complex patients). At the time of our study, the CTP program was not fully implemented in all parts of the ECH practice. The CTP was available to the downtown primary care internal medicine service, but the remaining three sites and family medicine service did not have CTP coverage.

\section{Participants}

All the subjects were hospitalized patients who were discharged from the hospital to the community. A total of 40 subjects over 60 years of age were recruited, including 20 patients that used the CTP and 20 patients who were discharged from the hospital without using the CTP. The study patients were recruited shortly after discharge from the hospital. Patients were eligible for the study if they were medically complex individuals with a high risk of readmission based upon their Elder Risk Assessment (ERA) score, a risk stratification instrument correlated with subsequent readmissions. ${ }^{16}$ ERA scores above 16 placed the patients in the top $10 \%$ for risk of hospital admission. The ERA index predicts hospital stays and ER visits ${ }^{16}$ and also predicts mortality and nursing home placement. ${ }^{17}$ ERA scores were derived electronically and were continuously updated in the Mayo Clinic electronic medical record (EMR). The ERA score is used clinically on all patients within Mayo Clinic to determine eligibility for case management. The ERA score heavily weights previous hospitalization (days) and age over 60. It also takes into consideration comorbid medical status. ${ }^{16}$ Participants in the CTP interventional arm were drawn from sections of the ECH practice currently using the CTP. The patients in the usual care (UC) group had the same ERA scores but were not enrolled in the CTP. The exclusion criteria included patients discharged 
to a nursing home, those diagnosed with a terminal illness or dementia, and those enrolled in hospice care.

\section{Recruitment}

The ECH staff reviewed the Mayo Clinic EMR ERA scores to identify patients who were eligible for CTP. The CTP RN case manager then provided the study coordinator with a list of potential participants for the study. Eligible patients not enrolled in CTP were recruited in a similar fashion. Each subject was reimbursed $\$ 25$ for their time upon completion of the study.

\section{Care transition program}

The CTP team made initial contact with patients in the hospital. The CTP team included the NP, case manager $\mathrm{RN}$, the primary physician, and consulting geriatrician. If a patient was amenable to home visits, an NP visited the patient at home within 1 to 3 days after discharge. The initial visit included a review of medications, the hospital admission including the discharge summary, ongoing symptoms, and upcoming tests and appointments. This assessment also included a functional assessment, symptom assessment, and cognitive evaluation. The goals of care and advanced care planning are critical aspects of the program. After the initial visit, the NP made subsequent home or telephone visits, as required. A case manager who was an RN communicated weekly with CTP patients by phone. The RN also triaged patient phone calls, if symptoms arose. All patients in the CTP remained under care for a minimum of 30 days.

\section{Usual care}

All subjects in both the UC and CTP groups received UC. UC involved a comprehensive review of the medications by hospital consultant pharmacists and medication reconciliation by the discharging nursing team. All patients received a hospital discharge summary prior to their discharge from the hospital. Primary care physicians were notified of admissions and discharges from the hospital and had access to all notes, labs, upcoming tests, and the discharge summary, via the EMR. All patients received a phone call from an $\mathrm{RN}$ in the outpatient practice within 1 to 3 days after discharge. Follow-up primary care visits were coordinated if required.

\section{Primary outcomes}

The primary outcomes were hospital readmission and ER visits within 30 days of discharge. We sought to find the relationship between hospital readmission, ER visits and CTP involvement in medically complex adults. These out- comes were determined via subject self-reports and verified through chart abstraction.

\section{Secondary outcomes}

The secondary outcomes were functional status and QOL. Functional outcomes included grip strength and gait speed. The QOL was measured at baseline and at a 1-month follow-up using the Short Form $\left(\mathrm{SF}^{\circledR}\right)-12^{1}$ (QualityMetric, Inc, Lincoln, RI, USA), an internationally validated, 12-item health survey including both physical and mental subcategories.

\section{Predictors}

The initial data collected at baseline included demographic information, such as age, gender, marital status, race, ethnicity, and number of people within the home, as well as functional status, QOL, and comorbid health conditions as defined by the ERA score at study entry. We determined the functional status based on grip strength of the dominant hand (measured in pounds and converted to kilograms) using a dynamometer (Baseline ${ }^{\circledR}$ model, Fabrication Enterprise, Inc., Elmsford, NY, USA), and gait speed over six meters was measured and reported in seconds. Patients performed a self-assessment of their functional status using the Duke Activity Status Index (DASI). ${ }^{2}$ The QOL was determined using the SF-12. ${ }^{18}$ A higher score indicates better physical or mental functioning. To calculate the physical and mental SF-12 scores, the test items were scored and normalized. The possible SF-12 scores ranged from 0-100, with a mean score of 50 and a standard deviation (SD) of 10 in a representative sample of the US population. A mental or physical SF-12 score greater than 50 represents above-average health status compared with the US population. ${ }^{19}$

\section{Blinding}

The study was an unblinded prospective cohort trial because the participants and the investigators were obviously aware of the interventions. However, the analysis was performed in a blinded fashion.

\section{Analysis}

The initial characteristics of the demographics, comorbid illnesses, ERA scores, SF-12 scores (physical and mental), and DASI scores were compared between groups using a $t$-test for continuous variables and a Pearson Chi-square analysis for proportional variables. The primary outcomes of 30-day hospital readmissions and ER visits were compared with a time-to-event analysis (Cox proportional analysis). 
This analysis was unadjusted since there were no clinically significant differences between the two groups at baseline. The secondary evaluations used paired $t$-test scores for grip strength, gait speed, and QOL information.

Subject characteristics were summarized using mean $( \pm \mathrm{SD})$ and median (25-75 interquartile range) for continuous variable and frequency percentages for nominal variables. An exploratory analysis, to assess whether changes in the scores of the measures listed above differed within subjects, was performed using a paired $t$-test. In all cases, $P$-values of $<0.05$ were considered statistically significant.

\section{Power analysis}

This was a pilot cohort study to determine the variance between the CTP and UC. This study was not fully powered to determine a difference between the two groups. Using an approximate estimation of $20 \%$ hospital readmission in the UC group and an expected $30 \%$ reduction from the CTP group required 251 subjects in each group. Patient information was recorded in REDCap (hosted by Mayo Clinic clinical and translational science center) by the study team. REDCap is a secure data system that allows tracking of patient information. ${ }^{20}$ Analysis was performed using SAS 9.12 (SAS Institute, Cary, NC, USA).

\section{Results}

\section{Recruitment and patient characteristics}

We made 337 phone calls and found 61 patients (18\%) to be eligible. Of the 61 patients, 40 consented to enroll in the study and 36 completed the study. Full details are noted in

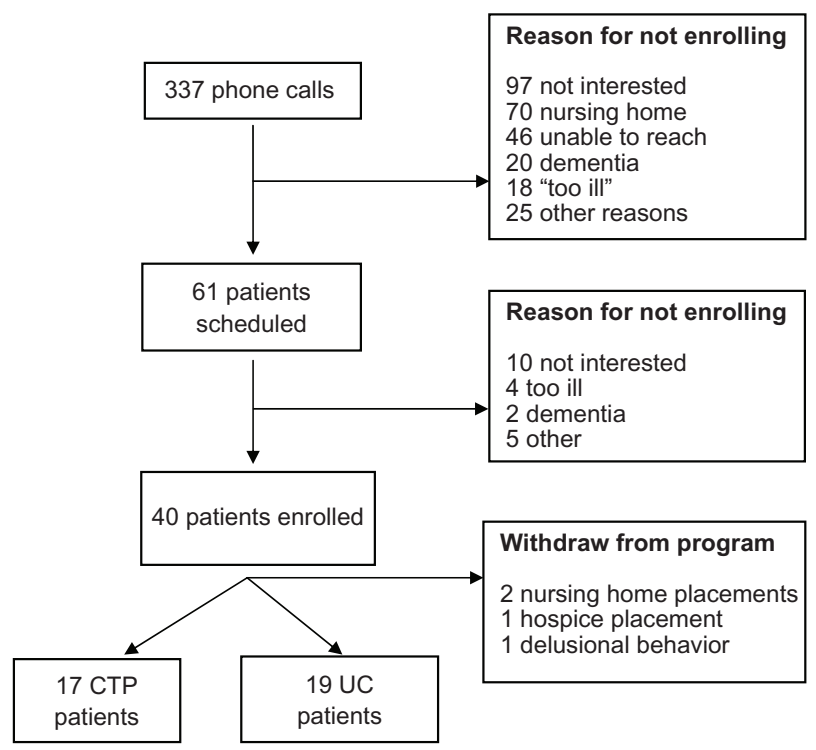

Figure I Enrollment of patients in the care transitions cohort study. Abbreviations: CTP, care transition program; UC, usual care.
Figure 1. The average age of the participants was 78.6 years ( \pm SD 9.4 years). Comparing the CTP group with the UC cohort, there were no systematic differences between the groups with regard to age, race, ethnicity, marital status, sex, weight, or DASI score. The patient characteristics, by group and for the overall cohort, are noted in Table 1. Based upon these characteristics, no adjustments were made to the analysis.

\section{Primary and secondary outcomes}

In comparing the CTP and UC patients, there was no difference in 30-day hospital readmission rates $(10.5 \%$ for UC patients versus no readmission for the CTP patients). There were no differences in ER visits for the CTP patients (11.8\%) compared with UC patients $(31.6 \%)(P=0.37)$. All the outcomes showed nonsignificant improvement for the CTP group compared with the UC group; this is shown in Table 2.

The secondary analysis of functional outcomes of grip strength and gait speed showed no differences between baseline and the 30-day follow-up, for the CTP and UC groups. The average time to walk six meters increased slightly for the CTP group and decreased slightly for the UC group; however, there was no within-group significance. The baseline gait speeds over 6 meters were 9.3 seconds (0.64 meters/second) for the CTP group versus $12.0 \mathrm{sec}-$ onds ( 0.50 meters/second) for the UC group. Grip strength declined slightly for both groups and was not significant. Physical and mental QOL scores were not different between the CTP group and UC ( $P=0.13$ and $P=0.06$ respectively). The within-group evaluations did show some significant differences. Physical QOL scores from the SF-12 showed improvement over 5 weeks for the CTP group, with a 6.7 point increase $(P<0.01)$. Mental QOL increased significantly for the UC group by 5.6 points $(P=0.04)$. Adjusting for multiple comparisons, the changes in mental QOL scores may be more of a borderline finding. Full findings are noted in Table 3 .

\section{Discussion}

In this pilot study of 36 patients, we found no difference in the number of 30-day hospital readmissions or ER visits for CTP patients compared with UC patients. However, we did see lower event rates for both 30-day readmissions and ER visits for the CTP group. We found a 10\% absolute decrease in the 30-day rehospitalization rate of CTP patients compared with UC patients. We also found an absolute decrease of over $20 \%$ in ER visits. Our power to detect a difference 
Table I Baselines and demographics of 36 patients in care transition and usual care

\begin{tabular}{|c|c|c|c|c|}
\hline & $\begin{array}{l}\text { CTP } \\
(n=17)\end{array}$ & $\begin{array}{l}\text { UC } \\
(n=19)\end{array}$ & $\begin{array}{l}\text { Total } \\
(n=36)\end{array}$ & $P$-value \\
\hline Subjects' gender & & & & $0.7 I^{\mathrm{a}}$ \\
\hline Male & $10(58.8 \%)$ & $10(52.6 \%)$ & $20(55.6 \%)$ & \\
\hline Female & $7(41.2 \%)$ & $9(47.4 \%)$ & $16(44.4 \%)$ & \\
\hline Subjects' marital status & & & & $0.89^{a}$ \\
\hline Never married & I (5.9\%) & I (5.3\%) & $2(5.6 \%)$ & \\
\hline Separated/divorced & $3(17.6 \%)$ & $4(21.1 \%)$ & 7 (19.4\%) & \\
\hline Widowed & $6(35.3 \%)$ & $5(26.3 \%)$ & II (30.6\%) & \\
\hline Married & 7 (4I.2\%) & $8(42.1 \%)$ & $15(41.7 \%)$ & \\
\hline Other & $0(0.0 \%)$ & I (5.3\%) & I (2.8\%) & \\
\hline \multicolumn{5}{|l|}{ Subjects' ethnicity } \\
\hline Non-Hispanic or non-Latino & $17(100.0 \%)$ & $19(100.0 \%)$ & $36(100.0 \%)$ & \\
\hline Subjects' race & & & & $0.28^{a}$ \\
\hline American Indian or Alaskan Native & I (5.9\%) & $0(0.0 \%)$ & I (2.8\%) & \\
\hline White & $16(94.1 \%)$ & $19(100.0 \%)$ & 35 (97.2\%) & \\
\hline Number of people in the same household & & & & $0.45^{\mathrm{a}}$ \\
\hline 0 & $9(52.9 \%)$ & $6(31.6 \%)$ & $15(4 \mid .7 \%)$ & \\
\hline I & $5(29.4 \%)$ & $9(47.4 \%)$ & $14(38.9 \%)$ & \\
\hline 2 & $3(17.6 \%)$ & $3(15.8 \%)$ & $6(16.7 \%)$ & \\
\hline 4 & $0(0.0 \%)$ & I (5.3\%) & I (2.8\%) & \\
\hline Subjects' weight (kg) & & & & $0.48^{\mathrm{b}}$ \\
\hline Mean (SD) & $86.7(29.3)$ & $78.4(19.7)$ & $82.3(24.7)$ & \\
\hline Median & 83.4 & 74.1 & 82.5 & \\
\hline QI, Q3 & $76.1,101.5$ & $59.1,100.3$ & $62.0,100.3$ & \\
\hline Range & $(42.2-168.9)$ & $(44.8-107.6)$ & $(42.2-168.9)$ & \\
\hline Subjects' age at time of consent & & & & $0.60^{\mathrm{b}}$ \\
\hline Mean (SD) & $79.6(6.6)$ & $77.8(11.5)$ & $78.6(9.4)$ & \\
\hline Median & 81.8 & 80.4 & 80.9 & \\
\hline QI, Q3 & $76.5,83.3$ & $69.0,88.3$ & $70.7,84.0$ & \\
\hline Range & $(64.7-91.1)$ & $(6 I .1-100.9)$ & $(61.1-100.9)$ & \\
\hline ERA score & & & & $0.53^{b}$ \\
\hline Mean (SD) & $18.5(2.3)$ & $19.1(3.1)$ & $18.8(2.7)$ & \\
\hline Median & 18.0 & 19.0 & 18.0 & \\
\hline QI, Q3 & $17.0,21.0$ & $16.0,20.0$ & $17.0,20.5$ & \\
\hline Range & $(16.0-24.0)$ & $(16.0-26.0)$ & $(16.0-26.0)$ & \\
\hline Duke activity status index & & & & $0.28^{b}$ \\
\hline Mean (SD) & $24.0(15.3)$ & $18.6(12.5)$ & 21.1 (13.9) & \\
\hline Median & 19.0 & 17.5 & 19.0 & \\
\hline QI, Q3 & $13.0,29.0$ & $10.0,21.0$ & II.0, 27.0 & \\
\hline Range & $(5.0-58.0)$ & $(2.0-45.0)$ & $(2.0-58.0)$ & \\
\hline
\end{tabular}

Notes: ${ }^{\mathrm{a} C h i}$-square test; ${ }^{\mathrm{b}}$-test.

Abbreviations: CTP, care transition program; ERA, Elder Risk Assessment; SD, standard deviation; UC, usual care.

was 0.09 in this pilot study. We would have needed a study of 251 patients in each intervention to have a power of 0.8 . While this pilot study did not detect a difference between the groups, we were encouraged that the results trended toward a reduction in 30-day hospital readmission. We are optimistic that further evaluations of our CTP in studies with larger numbers of patients may confirm a benefit to medically complex patients. We understand that one cannot make conclusions about the efficacy of the program based on this pilot data. The lack of efficacy may also reflect the variations in initial practice in this new model. There can
Table 2 30-day hospitalization and emergency room visits for 17 care transition patients and 19 usual care patients

\begin{tabular}{lllll}
\hline & $\begin{array}{l}\text { CTP } \\
(\mathbf{n}=17)\end{array}$ & $\begin{array}{l}\text { UC } \\
(\mathbf{n}=19)\end{array}$ & $\begin{array}{l}\text { Total } \\
(\mathbf{n}=36)\end{array}$ & P-value $^{\mathbf{a}}$ \\
\hline $\begin{array}{llll}\text { Inpatient } \\
\text { hospitalization }\end{array}$ & & & 0.17 \\
$\quad$ No & $17(100.0 \%)$ & $17(89.5 \%)$ & $34(94.4 \%)$ & \\
$\quad \begin{array}{l}\text { Yes } \\
\text { ER visit }\end{array}$ & $0(0.0 \%)$ & $2(10.5 \%)$ & $2(5.6 \%)$ & \\
$\quad$ No & $15(88.2 \%)$ & $13(68.4 \%)$ & $29(80.6 \%)$ & \\
Yes & $2(11.8 \%)$ & $6(31.6 \%)$ & $7(19.4 \%)$ & \\
\hline
\end{tabular}

Note: aCox proportional analysis.

Abbreviations: CTP, care transition program; ER, emergency room; UC, usual care. 
Table 3 Function and quality of life for 36 patients

\begin{tabular}{|c|c|c|c|c|c|c|}
\hline Functional outcomes & Baseline CTP & 5-week CTP & $P$-value & Baseline UC & 5-week UC & $P$-value \\
\hline Grip strength (kilograms) & & & 0.91 & & & 0.7 \\
\hline $\mathrm{N}$ & 16 & 15 & & 19 & 18 & \\
\hline Mean (SD) & $21.7(11.5)$ & $23.4(9.8)$ & & $21.4(10.5)$ & $22.3(9.8)$ & \\
\hline Median & 20 & 19 & & 18 & 20 & \\
\hline QI, Q3 & 14,32 & 15,36 & & 14,28 & 15,27 & \\
\hline Range & $(0.0-39.0)$ & $(11-39.0)$ & & $(7-44)$ & $(I I-45)$ & \\
\hline Gait speed (seconds) ${ }^{\mathrm{a}}$ & & & 0.95 & & & 0.56 \\
\hline $\mathrm{N}$ & 16 & 14 & & 18 & 18 & \\
\hline Mean (SD) & $9.3(4.5)$ & $9.9(4.8)$ & & $12.0(6.5)$ & $10.0(4.2)$ & \\
\hline Median & 9.2 & 8.5 & & 10.2 & 9.2 & \\
\hline QI, Q3 & $6.5,12.5$ & $6.5,11.8$ & & $6.7,16.0$ & $6.7,12.0$ & \\
\hline Range & $(0.0-17.9)$ & $(5.0-19.8)$ & & $(4.0-25.0)$ & $(4.3-20.0)$ & \\
\hline Quality of life $\mathrm{a}^{\mathrm{a}}$ & & & $<0.01$ & & & 0.19 \\
\hline $\mathrm{SF}^{\oplus}-12$ physical scale & 32.7 & 39.4 & & 31.9 & 34.9 & \\
\hline $\mathrm{SF}^{\circledR}-12$ mental scale & 56.2 & 56.2 & 0.99 & 51.6 & 57.2 & 0.04 \\
\hline Self-rated health ${ }^{\mathrm{b}}$ & & & 0.73 & & & 0.54 \\
\hline Missing & 0 & 0 & & 0 & I & \\
\hline Excellent & 0 & 0 & & I (5.3\%) & I (5.6\%) & \\
\hline Very good & $4(23.5 \%)$ & $3(17.6 \%)$ & & I (5.3\%) & I (5.6\%) & \\
\hline Good & 7 (4I.2\%) & $8(47.1 \%)$ & & 7 (36.8\%) & 8 (44.4\%) & \\
\hline Fair & 5 (29.4\%) & $6(35.3 \%)$ & & 7 (36.8\%) & 8 (44.4\%) & \\
\hline Poor & I (5.9\%) & $0(0.0 \%)$ & & 3 (15.8\%) & $0(0.0 \%)$ & \\
\hline
\end{tabular}

Notes: aPaired $t$-test; ${ }^{b}$ chi-square test.

Abbreviations: CTP, care transition program; SD, standard deviation; SF, Short Form; UC, usual care.

be wide variations in practice with the initiation of a new practice model..$^{21}$

Previous studies of CTP have shown encouraging results, thus our interest and investment in this clinical program. The clinical model that closely resembles our CTP program was described by Naylor et al, ${ }^{13}$ who used NPs to follow 239 patients with congestive heart failure (CHF) for 3 months after hospital discharge. The program used evidence-based CHF guidelines for NPs to follow, within the context of an interdisciplinary team. The group found a lower rehospitalization per patient year, 1.18 for CTP patients compared with 1.79 for UC patients $(P<0.001)$. Another NP transitional care model was evaluated in a study involving 172 matched pairs of at-risk older adults and also showed evidence of reduced hospital days and hospitalization. ${ }^{22}$ This second, common, transition program model emphasized provision of care to complex patients after discharge by RNs rather than NPs. One RN model showed a $48 \%$ reduction in 30-day readmissions compared with a UC group. ${ }^{12}$ Project RED used a standardized discharge process that resulted in a 30\% reduction in hospital utilization. ${ }^{11}$ Our CTP uses many of the best practices of project RED and other RN-based care transition programs and differs clinically because we used NPs to do home visits, RNs to take triage phone calls and hospital based discharge planning.
The secondary outcomes of gait speed and grip strength were not significantly different between the CTP and UC groups. The average gait speed was slow and below the conventional cutoff of $1.0 \mathrm{~meter} / \mathrm{second}$, currently a commonly associated risk factor for frailty. ${ }^{23}$ Functional evaluation as an outcome in care transitions has not been as well outlined as hospitalization in clinical trials. Self-reported dependency did not change in the original Naylor et al study of care transitions versus UC for CHF patients. ${ }^{13}$ Our CTP intervention did not specifically address sarcopenia; thus, the lack of change in gait speed and grip strength may reflect our lack of physical intervention. Interventions focused on increasing physical activity may improve aspects of functional status, like gait speed. ${ }^{24}$ The addition of an exercise program may enhance our clinical care transition interventions in the future.

In our study, the physical QOL for the CTP group improved significantly by 6.7 points. The mental QOL showed a borderline improvement for the UC group. The between-group comparisons were not significant. However, in previous studies, there was some evidence of improvement in QOL after interventions. In a study of 292 patients, a pre-post design revealed improvement in both the physical and emotional components of the $\mathrm{SF}^{\circledR}-36$ Health Survey (QualityMetric, Inc). ${ }^{25}$ In Naylor's group, there appeared to 
be some improvement in CHF QOL at 2 and 12 weeks, in the intervention group compared with the UC group. ${ }^{13}$ The physical QOL scores were low at baseline, with an average score more than 1.5 SDs below the norm. The improvement may reflect natural healing after hospitalization, or it may reflect better management by the CTP team. Thus, previously hospitalized patients may return to baseline health. Interestingly, in the UC group, there was only a 3-point improvement in the physical QOL scores. Further evaluations at longer follow-up may provide better information about the impact of the intervention on QOL.

The study has some inherent limitations common in cohort studies. One limitation involves the comparability between groups. It is possible that there were inherent differences between the groups that were not evaluated or adjusted at baseline. Although the potential for confounders exist, the major predictors for hospitalization are age, gender, and comorbid status; these are the bases for risk stratification instruments such as the $\mathrm{PRA}^{\mathrm{TM}}$ and other ambulatory care group instruments. ${ }^{26,27} \mathrm{We}$ evaluated these common risk factors, and they were comparable between the two groups. This was a small pilot study and the power to detect a difference was 0.09 , which limited our ability to detect a significant difference between groups. With $11 \%$ accrual of potential subjects, the individuals in the study may represent those feeling well enough to participate in the study (volunteer bias). Volunteer bias should not differentially affect the results between the groups. Volunteer bias may affect the ability to generalize the results beyond this group. We also excluded patients with dementia, who make up an important percentage of the population over sixty years.

\section{Conclusion}

This pilot study provides some opportunities to evaluate a CTP in a new and different medical system, using components from previous work. The pilot nature of the study design limits the ability to see a statistically significant difference; however, some general conclusions can be drawn. First, larger studies with similar care models will further our ability to see meaningful reductions in the number of 30-day rehospitalizations and reductions in the number of ER visits post discharge. Studies in the size range of 200-400 individuals would be required to see meaningful differences in the groups. Refinement of the clinical model will be needed to make continued incremental improvement in care. These refinements might include an exercise- or nutrition-focused intervention. Another important question involves the composition of the care transition team.
Should this include an NP team or an RN team? These questions will be critical as more health systems invest in care transition. Finally, other health outcomes, such as QOL, functional status, and symptom burden, will be critical measures as health systems invest in CTPs. We were encouraged that physical QOL did improve, even in this small pilot population.

\section{Disclosure}

Paul Takahashi serves as a medical advisor for Axial LLC (Raleigh, NC, USA). All other authors report no conflicts of interest.

\section{References}

1. Coleman EA, Min SJ, Chomiak A, Kramer AM. Posthospital care transitions: patterns, complications, and risk identification. Health Serv Res. 2004;39(5):1449-1465.

2. Feigenbaum P, Neuwirth E, Trowbridge L, et al. Factors contributing to all-cause 30-day readmissions: a structured case series across 18 hospitals. Med Care. 2012;50(7):599-605.

3. Naylor MD, Aiken LH, Kurtzman ET, Olds DM, Hirschman KB. The care span: the importance of transitional care in achieving health reform. Health Aff (Milkwood). 2011;30(4):746-754.

4. Whelan CT. The role of the hospitalist in quality improvement: systems for improving the care of patients with acute coronary syndrome. J Hosp Med. 2010;5 Suppl 4:S1-S7.

5. Cabana MD, Jee SH. Does continuity of care improve patient outcomes? J Fam Pract. 2004;53(12):974-980.

6. Misky GJ, Wald HL, Coleman EA. Post-hospitalization transitions: Examining the effects of timing of primary care provider follow-up. J Hosp Med. 2010;5(7):392-397.

7. Coleman EA, Boult C; American Geriatrics Society Health Care Systems Committee. Improving the quality of transitional care for persons with complex care needs. J Am Geriatr Soc. 2003;51(4): 556-557.

8. Foust JB, Naylor MD, Bixby MB, Ratcliffe SJ. Medication problems occurring at hospital discharge among older adults with heart failure. Res Gerontol Nurs. 2012;5(1):25-33.

9. Novak CJ, Hastanan S, Moradi M, Terry DF. Reducing unnecessary hospital readmissions: the pharmacist's role in care transitions. Consult Pharm. 2012;27(3):174-179.

10. Abrashkin KA, Cho HJ, Torgalkar S, Markoff B. Improving transitions of care from hospital to home: what works? Mt Sinai J Med. 2012;79(5):535-544.

11. Jack BW, Chetty VK, Anthony D, et al. A reengineered hospital discharge program to decrease rehospitalization: a randomized trial. Ann Iintern Med. 2009;150(3):178-187.

12. Coleman EA, Smith JD, Frank JC, Min SJ, Parry C, Kramer AM. Preparing patients and caregivers to participate in care delivered across settings: the Care Transitions Intervention. J Am Geriatr Soc. 2004;52(11):1817-1825.

13. Naylor MD, Brooten DA, Campbell RL, Maislin G, McCauley KM, Schwartz JS. Transitional care of older adults hospitalized with heart failure: a randomized, controlled trial. J Am Geriatr Soc. 2004;52(5): 675-684.

14. Brock J, Mitchell J, Irby K, et al; Care Transition Project Team. Association between quality improvement for care transitions in communities and rehospitalizations among Medicare beneficiaries. JAMA. 2013;309(4):381-391.

15. Hesselink G, Schoonhoven L, Barach $P$, et al. Improving patient handovers from hospital to primary care: a systematic review. Ann Intern Med. 2012;157(6):417-428. 
16. Crane SJ, Tung EE, Hanson GJ, Cha S, Chaudhry R, Takahashi PY. Use of an electronic administrative database to identify older community dwelling adults at high-risk for hospitalization or emergency department visits: the elders risk assessment index. BMC Health Ser Res. 2010; $10: 338$.

17. Takahashi PY, Tung EE, Crane SJ, Chaudhry R, Cha S, Hanson GJ. Use of the elderly risk assessment (ERA) index to predict 2-year mortality and nursing home placement among community dwelling older adults. Arch Gerontol Geriatr. 2012;54(1):34-38.

18. Gandek B, Ware JE, Aaronson NK, et al. Cross-validation of item selection and scoring for the SF-12 Health Survey in nine countries: results from the IQOLA Project. International Quality of Life Assessment. J Clin Epidemiol. 1998;51(11):1171-1178.

19. Ware J Jr, Kosinski M, Keller SD. A 12-item Short-Form Health Survey: construction of scales and preliminary tests of reliability and validity. Med Care. 1996;34(3):220-233.

20. Harris PA, Taylor R, Thielke R, Payne J, Gonzalez N, Conde JG. Research electronic data capture (REDCap) - a metadata-driven methodology and workflow process for providing translational research informatics support. J Biomed Inform. 2009;42(2):377-381.
21. Richards DA, Bower P, Pagel C, et al. Delivering stepped care: an analysis of implementation in routine practice. Implement Sci. 2012;7:3.

22. Naylor MD, Bowles KH, McCauley KM, et al. High-value transitional care: translation of research into practice. J Eval Clin Pract. Epub March 16, 2011.

23. Fielding RA, Vellas B, Evans WJ, et al. Sarcopenia: an undiagnosed condition in older adults. Current consensus definition: prevalence, etiology, and consequences. International Working Group on Sarcopenia. J Am Med Dir Assoc. 2011;12(4):249-256.

24. Morie M, Reid KF, Miciek R, et al. Habitual physical activity levels are associated with performance in measures of physical function and mobility in older men. J Am Geriatr Soc. 2010;58(9):1727-1733.

25. Watkins L, Hall C, Kring D. Hospital to home: a transition program for frail older adults. Prof Case Manag. 2012;17(3):117-123.

26. Boult C, Dowd B, McCaffrey D, Boult L, Hernandez R, Krulewitch H. Screening elders for risk of hospital admission. J Am Geriatr Soc. 1993;41(8):811-817.

27. Weiner JP, Starfield BH, Steinwachs DM, Mumford LM. Development and application of a population-oriented measure of ambulatory care case-mix. Med Care. 1991;29(5):452-472.
Clinical Interventions in Aging

\section{Publish your work in this journal}

Clinical Interventions in Aging is an international, peer-reviewed journal focusing on evidence-based reports on the value or lack thereof of treatments intended to prevent or delay the onset of maladaptive correlates of aging in human beings. This journal is indexed on PubMed Central, MedLine, the American Chemical Society's 'Chemical Abstracts

\section{Dovepress}

Service' (CAS), Scopus and the Elsevier Bibliographic databases. The manuscript management system is completely online and includes a very quick and fair peer-review system, which is all easy to use. Visit http://www.dovepress.com/testimonials.php to read real quotes from published authors. 